\title{
Datos sobre las comunidades ibéricas de Pinguiculion longifoliae F. Casas 1970 (Cl. Adiantetea capilli-veneris Br.-BI. in Br.-BI., Roussine \& Nègre 1952)
}

\author{
Manuel B. Crespo, Ma Ángeles Alonso \& Mario Martínez-Azorín
}

Dpto. de Ciencias Ambientales y Recursos Naturales, Universidad de Alicante, Apartado 99. E-03080 Alicante, España.

\section{Correspondencia \\ Manuel B. Crespo}

e-mail: crespo@ua.es

Recibido: 16 noviembre 2020

Aceptado: 19 noviembre 2020

Publicado on-line: 13 enero 2021

Editado por: Marta Recio

\begin{abstract}
Resumen
Se presentan nuevos datos sobre las comunidades rupícolas de la alianza Pinguiculion longifoliae en la Península Ibérica. Estos tipos de vegetación crecen en roquedos calcáreos y dolomíticos, particularmente en paredones y abrigos umbrosos, sobre suelos apenas desarrollados donde se forman tobas que permanecen húmedas y rezumantes durante gran parte del año, debido al afloramiento continuo de agua. Aunque hasta el momento se venía aceptando la existencia de cinco asociaciones en la Península lbérica, estudios recientes sobre la taxonomía de las especies rupícolas de Pinguicula en el este y sur peninsulares permite una nueva interpretación de estos frágiles hábitats. En la presente aportación se reconocen ocho asociaciones en la alianza, de las que una se describe como nueva (Eucladio verticillati-Pinguiculetum casperianae ass. nov.); se realiza una nueva combinación nomenclatural en el rango de asociación (Adianto capilli-veneris-Pinguiculetum dertosensis comb. nov.); y se corrige el nombre de otra asociación (Southbyo tophaceae-Pinguiculetum tejedensis nom. corr.). Para todas ellas se ofrecen datos sobre su sinonimia, composición florística habitual, ecología y corología, y se indica también su tipo nomenclatural (incluyendo la designación de un lectótipo para la AdiantoPinguiculetum dertosensis)
\end{abstract}

Palabras clave: Pinguiculion longifoliae, Adiantetea, vegetación rupícola, nomenclatura, sintaxonomía, Península Ibérica.

\begin{abstract}
New data on the Iberian plant communities of Pinguiculion longifoliae $F$. Casas 1970 ass. nov. (Class Adiantetea capilli veneris Br.-Bl. in Br.-Bl., Roussine \& Nègre 1952)

New data are presented on the rupicolous communities of Pinguiculion longifoliae, which occur in the Iberian Peninsula. They grow mostly on vertical, limestone or dolomitic cliffs, namely in shady rock crevices with scarcely developed soil, where tufa deposits are formed that remain sodden and dripping during most of the year due to water emergence. Although up to now the existence of five Iberian associations had been widely accepted, recent studies on the taxonomy of the rupicolous species of Pinguicula in the eastern and southern Iberian Peninsula allows a new interpretation of these fragile habitats. In this contribution, eight associations are recognised in the alliance, one of which is described as new (Eucladio verticillati-Pinguiculetum casperianae ass. nov.); a new nomenclatural combination is made at the association rank (Adianto capilliveneris-Pinguiculetum dertosensis comb. nov.); and a nomenclatural correction is made for another one (Southbyo tophaceae-Pinguiculetum tejedensis nom. corr.). For each accepted syntaxon, data on their synonymy, usual floristic composition, ecology and chorology are provided, and their nomenclatural type is indicated (including the designation of a lectotype for Adianto-Pinguiculetum dertosensis).
\end{abstract}

Key words: Pinguiculion longifoliae, Adiantetea, rupicolous vegetation, nomenclature, syntaxonomy, Iberian Peninsula.

\section{Introducción}

En el Mediterráneo occidental, las comunidades dominadas por especies del género Pinguicula L. (Lentibulariaceae) se desarrollan en dos tipos de hábitats bien diferenciados (cf. Crespo et al., 2019a): prados higroturbosos de cualquier naturaleza aunque con mayor frecuencia ácidos- (Ord. Caricetalia fuscae Koch $1926 \mathrm{em}$. Br.-BI. 1949, Cl. Scheuchzerio-Caricetea nigrae Tüxen 1937) y 
roquedos calcáreos donde se forman tobas calizas (All. Pinguiculion longifoliae F. Casas 1970, Ord. Adiantetalia capilli-veneris Br.-BI. ex Horvatić 1934, Cl. Adiantetea Br.-BI. in Br.-Bl., Roussine \& Nègre 1952).

Los recientes estudios sobre la taxonomía de las especies rupícolas de Pinguicula en el este y sur de la Península Ibérica (Crespo et al., 2019a, $2019 b, 2020$ ) han revelado la existencia de varias especies inéditas o minusvaloradas -a menudo, endemismos de distribución reducida-, que habían pasado desapercibidas al haberse confundido con otros congéneres, a los que en consecuencia se les atribuían distribuciones más amplias. Como resultado, el catálogo de especies ibéricas de este género ha pasado de 9 a 13 (cf. Crespo et al., 2020), habiéndose descrito como novedades $P$. saetabensis M.B. Crespo, Mart.-Azorín \& M.Á. Alonso -endemismo setabense, a menudo citado como $P$. mundi Blanca, Jamilena, Ruiz Rejón \& Reg. Zamora o $P$. vallisneriifolia Webb-, $P$. tejedensis M.B. Crespo, Mart.-Azorín, M.Á. Alonso \& L. Sáez -endemismo Granadino-Almijarense, atribuido a $P$. dertosensis (Cañig.) Mateo \& M.B. Crespo- y P. casperiana M.B. Crespo, Mart.-Azorín, M.Á. Alonso \& L. Sáez -endemismo lbérico Maestracense, identificado como $P$. mundi o $P$. dertosensis-. Además, se ha reivindicado la separación específica de $P$. submediterranea Blanca, Jamilena, Ruiz Rejón \& Reg. Zamora, endemismo Cazorlense, hasta ahora sinonimizada a P. dertosensis (cf. Blanca, 2001; Blanca et al., 1999).

Casi todas las especies antes mencionadas presentan su óptimo ecológico en las comunidades briocormofíticas de Pinguiculion longifoliae, que, como se ha dicho anteriormente, se desarrollan de ordinario en paredones y abrigos umbrosos de naturaleza calcárea o dolomítica, donde se forman tobas que permanecen húmedas y rezumantes durante gran parte del año, debido al afloramiento continuo de agua (cf. Rivas-Martínez et al., 2002). Esta alianza presenta una amplia distribución en las sierras calcáreas del Mediterráneo occidental y ha sido estudiada por Foucault (2015). En la Península lbérica se han reconocido únicamente cinco (cf. Rivas-Martínez et al., 2001) o seis (cf. Foucault, 2015) asociaciones, distribuidas principalmente por el norte y este del territorio.

En la presente contribución, sobre la base de la nueva interpretación taxonómica de Pinguicula en las sierras del este y sur de la Península Ibérica (cf. Crespo et al., 2019b, 2020), se presentan datos preliminares de ocho asociaciones ibéricas de Pinguiculion longifoliae, que representan hábitats de gran rareza y fragilidad, a menudo muy seriamente amenazados. En todos los casos se aportan datos sintéticos sobre su sinonimia, tipos nomenclaturales, composición florística, sinecología y sincorología. De ellas, una asociación se describe como nueva; una subasociación se eleva al rango de asociación y se le designa un lectótipo; y, además, se corrige el nombre de otra de ellas.

\section{Material y métodos}

La caracterización bioclimática y biogeográfica de los sintaxones mencionados se ajusta a la tipología de Rivas-Martínez (2007). Las autorías de los sintaxones corresponden a las recogidas por RivasMartínez et al. $(2001,2002)$ y siguen la propuesta de Izco (in Rivas-Martínez et al., 2002). La tipificación de los sintaxones se ajusta al articulado del Código Internacional de Nomenclatura Fitosociológica (Theurillat et al., 2020), citándose los artículos convenientes entre paréntesis, cuando es necesario.

\section{Resultados y discusión}

1. Adianto capilli-veneris-Pinguiculetum longifoliae F. Casas 1970 [Ars Pharm. 11: 278; lectotypus (designado por Fernández Casas \& Benito Alonso, 1999: 114): tabla 6, inv. 1 (Huesca. Foradada del Toscar, Morillo de Liena, valle del río Esera, Pirineos)] Syn.: Pinguiculo longifoliae-Adiantetum capilli-veneris Rivas-Martínez, Báscones, T.E. Díaz, FernándezGonzález \& Loidi 1991 [Itinera Geobot. 5: 404-406; tabla 78; sin holótipo], nom. inval. (Art. 5)

Composición florística habitual. Pinguicula longifolia Ramond. ex DC., Adiantum capillus-veneris L., Eucladium verticillatum (Brid.) Bruch \& Schimp., Hypericum nummularium L., Hymenostylium recurvirostre (Hedw.) Dixon, Hieracium phlomoides Froel. in DC. (dif. transgr, de Saxifragion mediae Br.-BI. in Meier \& Br.-BI. 1934), Carex brachystachys Schrank (transgr. de Violo biflorae-Cystopteridion alpinae F. Casas 1970), Molinia caerulea (L.) Moench subsp. caerulea, Petrocoptis crassifolia (dif. transgr. de Valeriano longiflorae-Petrocoptidion F. Casas 1972), Saxifraga aizoides L. (dif. transgr. de Palustriellion commutatae Koch 1928 nom. mut.).

Sinecología y sincorología. Sólo se conoce de los Pirineos Centrales, en la provincia de Huesca, con óptimo en territorios mesotemplados (cf. Fernández Casas, 1970; Rivas-Martínez et al., 1991; FernándezCasas \& Benito Alonso, 1999); cabe mencionar que las referencias a $P$. longifolia y a esta asociación en Navarra, de Rivas-Martínez et al., 1991), no corresponden a dicha especie (cf. Blanca, 2001). Por tanto, esta asociación puede considerarse endémica del subsector Prepirenaico Aragonés, del Sector Prepirenaico (subprovincia Pirenaica, provincia Pirenaico-Cevenense, Subregión Alpino-Caucásica, Región Eurosiberiana).

2. Hyperico nummularii-Pinguiculetum coenocantabricae T.E. Díaz, Guerra \& Nieto 1982 corr. RivasMartínez, T.E. Díaz, F. Prieto, Loidi \& Penas 1984 [Vegetación Picos de Europa: 157].

Syn.: Hyperico-Pinguiculetum grandiflorae T.E. Díaz, Guerra \& Nieto 1982 [Anales Jard. Bot. Madrid 38(2): 502; holotypus: tabla 2, inv. 4 (Asturias. Amieva, Desfiladero de los Beyos, Picos de Europa)] 
Composición florística habitual. Pinguicula grandiflora subsp. coenocantabrica Rivas Mart., T.E. Díaz, Fern. Prieto, Loidi \& Penas, Adiantum capillusveneris, Eucladium verticillatum, Hymenostylium recurvirostre, Hypericum nummularium, Molinia caerulea, Petrocoptis glaucifolia (Lag.) Boiss. (dif. transgr. de Petrocoptidion glaucifoliae (P. Fernández, Penas \& T.E. Díaz 1983) Rivas-Martínez, Cantó \& Izco in Rivas-Martínez et al. 2001), Campanula rotundifolia L. subsp. legionensis (Pau) Laínz (dif. transgr. de Saxifragion trifurcato-canaliculatae RivasMartínez ex Rivas-Martínez, Izco \& Costa 1971); en zonas con mayor escorrentía se enriquece con Palustriella commutata (Hedw.) Ochyra.

Sinecología y sincorología. Sólo se conoce de los afloramientos calcáreos de los Picos de Europa, desde Covadonga y el Desfiladero del Cares (Asturias) hasta el Desfiladero de la Hermida y Venta de Fresnedo (Cantabria), en áreas termotempladas y mesotempladas (Díaz et al., 1982; Rivas-Martínez et al., 1984). Por tanto, resulta endémica del distrito Picoeuropeano, subsector Picoeuropeano-Ubiñense Septentrional, del Sector Picoeuropeano-Ubiñense (subprovincia Orocantábrica, provincia Atlántica Europea, Subregión Atlántico-Centroeuropea, Región Eurosiberiana).

\section{Adianto capilli-veneris-Pinguiculetum derto-} sensis (O. Bolòs 1967) Deil ex M.B. Crespo, M.Á. Alonso \& Mart.-Azorín, comb. \& stat. nov. (Art. 27d \& 51)

Basion.: Eucladio-Adiantetum subass. Pinguiculetosum dertosensis O. Bolòs 1967 [Mem. Real Acad. $\mathrm{Ci}$. Artes 38(1): 19; lectotypus (hic designatus): Tabla 8, inv. 2 (Castellón. Bellestar, Vall del Salt, sobre el vivero forestal de La Tenalla)]

Composición florística habitual. Pinguicula dertosensis, Adiantum capillus-veneris, Samolus valerandi L., Eucladium verticillatum, Pellia endiviifolia (Dicks.) Dumort. (incl. P. fabbroniana Raddi), Molinia caerulea subsp. arundinacea (Schrank) K. Richt., Schoenus nigricans L., Potentilla caulescens (transgr. de Potentilletalia caulescentis $\mathrm{Br}$.-BI. in Br.-BI. \& Jenny 1926), Valeriana tripteris L. subsp. tarraconensis (Pau) Devesa et al. (dif. transgr. de Saxifragion mediae), Salix tarraconensis Pau (dif. transgr. de Saxifragion mediae).

Sinecología y sincorología. Sólo se conoce de los Puertos de Tortosa y Beceite, en la zona de confluencia de las provincias de Castellón, Tarragona y Teruel (Bolòs, 1967; Royo, 2006), en territorios mesomediterráneos y supramediterráneos, bajo ombrótipo subhúmedo. Resulta endémica del distrito Puertobeceitano-Morellano, subsector Catalánido Occidental, del Sector Valenciano-Tarraconense (subprovincia Valenciana, provincia CatalanoProvenzal-Balear).
Observaciones. Foucault (2015) recogió el nombre de la presente asociación a través de una combinación atribuida a Deil (1989). Sin embargo, este último autor se refirió a ésta como "AdiantoPinguiculetum dertosensis stat. nov. prov. (= Eucladio-Adiantetum subass. pinguiculetosum dertosensis Bolòs 1967)", por lo que no puede tenerse por combinación válidamente publicada en 1989, al haberse establecido explícitamente como un nombre en un rango nuevo provisional (Art. 3b), basado en la subasociación puertobeceitana de Bolòs (1967). Por ello, se valida aquí dicha combinación en el rango de asociación y se atribuye al Prof. Ulrich Deil la autoría, de acuerdo con los Art. $27 d \& 51$.

4. Eucladio verticillati-Pinguiculetum casperianae M.B. Crespo, M.Á. Alonso \& Mart.-Azorín ass. nov. hoc loco [Tabla 1; holotypus: inv. 1 (Cuenca. Fuertescusa, hacia Poyatos, pr. Fuente de las Mujeres, río Escabas)]

Composición florística habitual. Pinguicula casperiana, Adiantum capillus-veneris, Eucladium verticillatum, Molinia caerulea subsp. arundinacea, Moehringia intricata Willk. subsp. castellana J.M. Monts. (dif. transgr. de Sarcocapnion enneaphyllae) y Antirrhinum pulverulentum Lázaro Ibiza (dif. transgr. de Jasionion foliosae O. Bolòs 1957).

Diagnosis. Comunidad de escasa diversidad, dominada por el endemismo oreoconquense Pinguicula casperiana ( $P$. mundi sensu auct.; $P$. dertosensis sensu auct.), al que unen de manera habitual y constante Eucladium verticillatum, Moehringia intricata subsp. castellana J.M. Monts. (dif. transgr.) y Potentilla caulescens (transgr.); con menor frecuencia participan Adiantum capillusveneris y Molinia caerulea subsp. arundinacea.

Merecen especial mención algunas especies transgresivas, de óptimo en comunidades catenalmente vecinas (Sarcocapnion enneaphyllae F. Casas 1972 y Jasionion foliosae O. Bolòs 1958), como es el caso de Antirrhinum pulverulentum, Rhamnus pumila Turra, Globularia repens subsp. borgiae G. López, Sarcocapnos enneaphylla (L.) DC. - Moehringia intricata subsp. castellana, que no suelen faltar en los roquedos del subsector Oreoconquense $y$, por tanto, pueden servir como diferenciales transgresivas con carácter local. Todos estos taxones, en conjunto, refuerzan el carácter lbérico Maestracense de la nueva asociación.

Como en otras comunidades de la alianza (Tabla 2), en la Eucladio-Pinguiculetum casperianae resulta variable el recubrimiento de los musgos y hepáticas, que aumenta con la constancia y caudal de la escorrentía. Los briófitos más corrientes son Eucladium verticillatum, Pellia endiviifolia, Philonotis calcarea (Bruch \& Schimp.) Schimp. y Porella platyphylla, acompañados a veces por Jungermannia atrovirens Dumort., Hymenostylium recurvirostre o Pseudoleskeella catenulata (Schrad.) Kindb., y a 
menudo complementados con mantos más o menos extensos de cianobacterias (Nostoc sp.).

Sinecología. La nueva asociación ocupa abrigos y paredones calcáreos (de ordinario, calizas y dolomías cretácicas y jurásicas), más o menos extraplomados. Se instala en grietas y oquedades por donde rezuma agua cargada de carbonato cálcico, que precipita formando tobas esponjosas que retienen cierta humedad, al menos desde mediados del otoño hasta finales de la primavera. Esta asociación se encuentra a baja y media altitud en la Serranía de Cuenca, entre 800 y 1600 m de elevación, en ambientes mesomediterráneos y supramediterráneos, bajo ombrótipo subhúmedo (a veces, húmedo localmente), de marcado carácter continental.

Tabla 1. Eucladio verticillati-Pinguiculetum casperianae M.B. Crespo, M.Á. Alonso \& Mart.-Azorín ass. nov. (Adiantetea capilli-veneris, Adiantetalia capilli-veneris, Pinguiculion longifoliae).

Table 1. Eucladio verticillati-Pinguiculetum casperianae M.B. Crespo, M.Á. Alonso \& Mart.-Azorín ass. nov. (Adiantetea capilli-veneris, Adiantetalia capilli-veneris, Pinguiculion longifoliae).

Eucladio verticillati-Pinguiculetum casperianae M.B. Crespo, M.Á. Alonso \& Mart.-Azorín ass. nov. (Adiantetea capilli-veneris, Adiantetalia capilli-veneris, Pinguiculion longifoliae)

\begin{tabular}{|c|c|c|c|c|c|c|c|}
\hline № de inventario & 1 & 2 & 3 & 4 & 5 & 6 & Índice \\
\hline Superficie $\left(m^{2}\right)$ & 4 & 5 & 4 & 3 & 4 & 2 & \\
\hline Recubrimiento (\%) & 95 & 90 & 90 & 85 & 90 & 100 & \\
\hline Elevación (m.s.n.m) & 980 & 1162 & 1162 & 1150 & 1300 & 1005 & \\
\hline Inclinación/orientación & $95^{\circ} \mathrm{N}$ & $90^{\circ} \mathrm{NE}$ & $90^{\circ} \mathrm{N}$ & $85^{\circ} \mathrm{NW}$ & $90^{\circ} \mathrm{NE}$ & $90^{\circ} \mathrm{NW}$ & \\
\hline \multicolumn{8}{|l|}{ Combinación característica: } \\
\hline Pinguicula casperiana & 5 & 5 & 4 & 4 & 4 & 2 & V \\
\hline Eucladium verticillatum & 2 & 3 & 2 & 1 & 1 & 5 & V \\
\hline Moehringia castellana (dif. tr.) & 1 & + & + & + & + & . & V \\
\hline Philonotis calcarea & + & 1 & + & . & . & 1 & IV \\
\hline Adiantum capillus-veneris & + & . & 1 & . & . & 2 & III \\
\hline Porella platyphylla & + & . & 1 & . & . & + & III \\
\hline Antirrhinum pulverulentum (dif. tr.) & + & . & . & + & + & . & III \\
\hline Jungermannia atrovirens & . & + & . & . & + & . & III \\
\hline Hymenostylium recurvirostre & + & . & 1 & . & . & . & II \\
\hline Pseudoleskeella catenulata & . & 1 & . & . & . & . & 1 \\
\hline \multicolumn{8}{|l|}{ Compañeras: } \\
\hline Potentilla caulescens & + & 1 & 1 & + & + & + & V \\
\hline Molinia caerulea subsp. arundinacea & + & 1 & + & + & & 1 & V \\
\hline Rhamnus pumila & + & + & . & + & + & . & IV \\
\hline Sarcocapnos enneaphylla & . & + & . & + & . & + & III \\
\hline Chaenorhinum crassifolium & . & + & . & + & . & . & II \\
\hline Globularia repens subsp. borgiae & . & . & . & . & + & . & I \\
\hline
\end{tabular}

Localidades (todas de la provincia de Cuenca, inventariadas el 29-mayo-2017): 1. Fuertescusa, hacia Poyatos, pr. Fuente de las Mujeres, río Escabas, 30 TWK738773 (holotypus). 2-3. Poyatos, Hoz de Beteta, pr. Fuente de los Tilos, 30TWK736890. 4. Poyatos, Hoz de Beteta, Cueva de la Ramera, 30TWK732883. 5. Uña, el Escalerón, 30TWK886534. 6. Poyatos, cueva del Barranco Malo, 30TWK770758.

Sincorología. Se conoce de amplias zonas del este y nordeste de la provincia de Cuenca, penetrando en las áreas limítrofes de Guadalajara, en el Alto Tajo. Por tanto, resulta endémica del Subsector 
Oreoconquense del Sector Ibérico Maestracense (Subprovincia Oroibérica, Provincia Mediterránea Ibérica Central).

Sintopografía. La Eucladio-Pinguiculetum casperianae contacta microtopográficamente de manera natural con las comunidades espeluncícolas de Chaenorhino crassifoliae-Sarcocapnetum enneaphyllae Rivas-Martínez \& G. López in G. López 1978 y con las comunidades rupícolas casmofíticas de Antirrhinetum pulverulenti F. Casas 1974, hacia los ecótopos más xéricos, donde no rezuma agua. No suelen formarse juncales o juncal-brezales al pie de estos abrigos y paredones oreoconquenses, como ocurre en otras comunidades de áreas más bajas 0 térmicas.

5. Adianto capilli-veneris-Pinguiculetum saetabensis M.B. Crespo, M.Á. Alonso, J.E. Oltra \& Mart.-
Azorín 2019 [Flora Montiber. 75: 8, holotypus: tabla 1, inv. 1 (Valencia. Enguera, barranco de la Hoz)].

Composición florística habitual. Pinguicula saetabensis, Adiantum capillus-veneris, Eucladium verticillatum, Riccia cavernosa Hoffm., Molinia caerulea subsp. arundinacea, Schoenus nigricans, Erica terminalis Salisb., Chaenorhinum tenellum (Cav.) Lange (dif. transgr. de Sarcocapnion pulcherrimae F. Casas 1972 corr. Rivas-Martínez, Cantó \& Izco 2002).

Sinecología y sincorología. Sólo se conoce de escasos puntos de la Sierra de Enguera, al sudoeste de la provincia de Valencia, en enclaves termomediterráneos, bajo ombrótipo subhúmedo (localmente, al menos) (Crespo et al., 2019a). Endémica del distrito Ayorano-Cofrentino, subsector Cofrentino-

Tabla 2. Cuadro sinóptico de las comunidades ibéricas de la alianza Pinguiculion longifoliae.

Table 2. Summary of the Iberian communities of the alliance Pinguiculion longifoliae.

\begin{tabular}{|c|c|c|c|c|c|c|c|c|}
\hline № orden de asociaciones & 1 & 2 & 3 & 4 & 5 & 6 & 7 & 8 \\
\hline № de inventarios & 8 & 9 & 7 & 6 & 6 & 4 & 7 & 3 \\
\hline Pinguicula longifolia & V & . & . & . & . & . & . & . \\
\hline Hieracium phlomoides & IV & & & & & & & \\
\hline Saxifraga aizoides & II & . & . & . & . & . & . & . \\
\hline Petrocoptis crassifolia & II & . & . & . & . & . & . & . \\
\hline Pinguicula coenocantabrica & . & $\mathrm{V}$ & . & . & . & . & . & . \\
\hline Campanula rotundifolia subsp. legionensis & . & IV & . & . & . & . & . & . \\
\hline Petrocoptis glaucifolia & . & III & . & . & . & . & . & . \\
\hline Pinguicula dertosensis & . & . & $\mathrm{V}$ & . & . & . & . & . \\
\hline Pinguicula casperiana & . & . & . & $\mathrm{V}$ & . & . & . & . \\
\hline Moehringia castellana & . & . & . & V & . & . & . & . \\
\hline Jungermannia atrovirens & . & . & . & III & . & . & . & . \\
\hline Pinguicula saetabensis & . & . & . & . & $\mathrm{V}$ & . & . & . \\
\hline Erica terminalis & . & . & . & . & $\mathrm{V}$ & . & . & . \\
\hline Chaenorhinum tenellum & . & . & . & . & IV & . & . & . \\
\hline Riccia cavernosa & . & . & . & . & $\mathrm{I}$ & . & . & . \\
\hline Pinguicula mundi & . & . & . & . & . & 4 & . & . \\
\hline Gymnostomum calcareum & . & . & . & . & . & 2 & . & . \\
\hline Pinguicula vallisneriifolia & . & . & . & . & . & . & $\mathrm{V}$ & . \\
\hline Hypericum caprifolium & . & . & . & . & . & . & III & . \\
\hline Jasione foliosa subsp. minuta & . & . & . & . & . & . & 1 & . \\
\hline Pinguicula tejedensis & . & . & . & . & . & . & . & 3 \\
\hline Southbya tophacea & . & . & . & . & & . & . & 2 \\
\hline \multicolumn{9}{|l|}{ Características de unidades superiores: } \\
\hline Adiantum capillus-veneris & V & $\mathrm{V}$ & V & III & IV & 3 & V & 1 \\
\hline Eucladium verticillatum & . & $\mathrm{V}$ & III & . & II & 4 & $\mathrm{~V}$ & 3 \\
\hline Pellia endiviifolia & & $\mathrm{V}$ & . & i & & 1 & i & . \\
\hline Hymenostylium recurvirostre & I & III & . & II & . & . & . & . \\
\hline Hypericum nummularium & IV & IV & . & . & . & . & . & . \\
\hline Philonotis calcarea & . & . & . & IV & . & . & . & . \\
\hline Pseudoleskeella catenulata & . & . & . & I & . & . & . & . \\
\hline \multicolumn{9}{|l|}{ Acompañantes: } \\
\hline Potentilla caulescens & . & . & II & V & 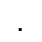 & 1 & IV & . \\
\hline Molinia caerulea subsp. arundinacea & . & . & V & V & I & . & . & . \\
\hline Rhamnus pumila & . & . & . & IV & . & . & . & . \\
\hline Sarcocapnos enneaphylla & . & . & . & III & & . & . & . \\
\hline Sarcocapnos saetabensis &. & . & . & . & 1 & . & . & . \\
\hline Globularia repens subsp. borgiae & . & . & . & I & & . & . & . \\
\hline
\end{tabular}

Asociaciones: 1. Adianto capilli-veneris-Pinguiculetum longifoliae (Fernández Casas, 1970, Tabla 6). 2. Hyperico nummularii-Pinguiculetum coenocantabricae (Díaz et al., 1982, Tabla 2). 3. Adianto capilli-venerisPinguiculetum dertosensis (Royo, 2006, Tabla 10, inv. 6-12). 4. Eucladio verticillati-Pinguiculetum casperianae (Tabla 1). 5. Adianto capilli-veneris-Pinguiculetum saetabensis (Crespo et al., 2019a, Tabla 1). 6. Eucladio verticillati-Pinguiculetum mundi (Díaz et al., 1982, Tabla 1, inv. 5-8). 7. Pinguiculetum vallisneriifoliae (Gómez Mercado, 2011, Tabla 11). 8. Southbyo tophaceae-Pinguiculetum tejedensis (Díaz et al., 1982, Tabla 1, inv. 1-3). 
Villenense, del Sector Setabense (subprovincia Valenciana, provincia Catalano-Provenzal-Balear).

6. Eucladio verticillati-Pinguiculetum mundi T.E. Díaz, Guerra \& Nieto 1982 corr. Asensi \& Díez Garretas in Rivas-Martínez, T.E. Díaz, FernándezGonzález, Izco, Loidi, Lousã \& Penas 2002 [Itinera Geobot. 15(1-2): 234]

Syn.: Eucladio-Pinguiculetum vallisneriifoliae T.E. Díaz, Guerra \& Nieto 1982 [Anales Jard. Bot. Madrid 38(2): 498; holotypus: tabla 1, inv. 5 (Albacete. Riópar, nacimiento del Río Mundo, Sierra de Alcaraz)]

Composición florística habitual. Pinguicula mundi, Adiantum capillus-veneris, Eucladium verticillatum, Pellia endiviifolia, Gymnostomum calcareum Nees \& Hornsch., Molinia caerulea subsp. arundinacea, Hypericum caprifolium Boiss., Valeriana tuberosa L., Teucrium rotundifolium Schreb. (dif. transgr. de Campanulion mollis Martínez-Parras \& Peinado 1990 nom. mut.); en zonas con escorrentía más acusada se enriquece con Cratoneuron filicinum (Hedw.) Roth.

Sinecología y sincorología. Sólo se conoce de los Calares del Río Mundo y de la Sima, en la provincia de Albacete, en territorios supramediterráneos (con irradiaciones mesomediterráneas), bajo ombrótipo subhúmedo (a veces, localmente húmedo) (cf. Díaz et al., 1982; Sánchez Gómez et al., 1997; Vera, 2015). Por tanto, resulta endémica del distrito Serrano-Alcaraceño, subsector Subbético Oriental, del Sector Subbético (provincia Bética).

7. Pinguiculetum vallisnerifoliae Heywood ex Varo \& F. Casas 1970 [Ars. Pharm. 11: 519; holotypus: inventario único (Jaén. Cazorla, Chorradillo de la Magdalena, a la sombra de una cueva), tomado de Heywood in Anales Jard. Bot. Madrid 11(2): 463-481, inv. 2 de la única tabla (1954)]

Composición florística habitual. Pinguicula vallisneriifolia, Adiantum capillus-veneris, Eucladium verticillatum, Molinia caerulea subsp. arundinacea, Schoenus nigricans, Hypericum caprifolium, Chaenorhinum villosum Lange (dif. transgr. de Asplenietea), Jasione foliosa subsp. minuta (C. Agardh ex Schult.) Font Quer (dif. transgr. de Saxifragion camposii Cuatrecasas ex Quézel 1953); Teucrium rotundifolium (dif. transgr.), Sarcocapnos baetica (Boiss. \& Reut.) Nyman (dif. transgr. de Sarcocapnion pulcherrimae); en zonas con escorrentía más acusada se enriquece con Cratoneuron filicinum.

Sinecología y sincorología. Crece en las sierras subbéticas de Cazorla y Segura (Jaén), en la zona limítrofe del río Tus (Albacete), con localidades disyuntas en la Sierra de Cázulas (Granada) y en Tous (Valencia), con óptimo en territorios termomediterráneos y mesomediterráneos, bajo ombrótipo subhúmedo (a veces localmente húmedo) (cf. Heywood, 1953; Varo \& Fernández-Casas, 1970; Gómez-Mercado, 2011; Pérez-Latorre et al., 2004;
Crespo et al., 2019a, 2019b). Por tanto, resulta casi endémica del subsector Cazorlense, del Sector Subbético (provincia Bética), con irradiaciones al subsector Almijarense, sector Granadino-Almijarense de la misma provincia biogeográfica, y con una localidad disyunta en el subsector CofrentinoVillenense, del Sector Setabense (subprovincia Valenciana, provincia Catalano-Provenzal-Balear).

\section{Southbyo tophaceae-Pinguiculetum tejedensis}

Asensi \& Díez Garretas in Rivas-Martínez, T.E. Díaz, Fernández-González, Izco, Loidi, Lousã \& Penas 2002 corr. M.B. Crespo, M.Á. Alonso \& Mart.-Azorín, nom. corr. hoc loco (Art. 43)

Syn.: Southbyo tophaceae-Pinguiculetum dertosensis Asensi \& Díez Garretas in Rivas-Martínez et al. 2002 [Itinera Geobot. 15(1-2): 208; holotypus: inventario único (Granada. Alhama, Salto del Caballo, $\mathrm{S}^{\mathrm{a}}$ de Tejeda; transcrito de Díaz González et al. (1982)]

Composición florística habitual. Pinguicula tejedensis, Adiantum capillus-veneris, Eucladium verticillatum, Southbya tophacea, Gymnostomum calcareum, Pellia endiviifolia, Hieracium texedense Pau (dif. transgr. de Saxifragion camposii), Jasione foliosa subsp. minuta (C. Agardh ex Schult.) Font Quer (dif. transgr. de Saxifragion camposii), Moehringia intricata subsp. tejedensis (Huter, Porta \& Rigo ex Willk.) J.M. Monts. (dif. transgr. de Sarcocapnion pulcherrimae), Brachypodium boissieri Nyman (dif. transgr. de Triseto velutini-Brachypodion boissieri Rivas-Martínez, Molero \& Pérez-Raya in Rivas-Martínez et al. 2001); en zonas con escorrentía más acusada se enriquece con Cratoneuron filicinum (Hedw.) Roth.

Sinecología y sincorología. Sólo se conoce de las Sierras de Tejeda y Almijara, en la provincia de Granada, con óptimo en territorios supramediterráneos (con irradiaciones hacia enclaves mesomediterráneos), bajo ombrótipo subhúmedo (Mota et al., 1991; Asensi \& Díez Garretas in Rivas-Martínez et al., 2002; Pérez Latorre et al., 2004). Es endémica del subsector Almijarense, Sector Granadino-Almijarense (provincia Bética).

Observaciones. Esta asociación fue descrita inicialmente con el nombre Southbyo tophaceaePinguiculetum dertosensis por Asensi \& Díez Garretas (in Rivas-Martínez et al., 2002), a partir de un solo inventario (holótipo) tomado en la Sierra de Tejeda, que había sido publicado previamente por Díaz et al. (1982) y atribuido por estos últimos autores a la "Eucladio-Pinguiculetum vallisneriifoliae" (nombre que fue posteriormente corregido a EucladioPinguiculetum mundi, como se ha dicho con anterioridad). Sin embargo, dado que la planta tejedense corresponde realmente a Pinguicula tejedensis (Crespo et al., 2020) y no a $P$. vallisneriifolia, es necesario corregir aquí el nombre original del sintaxon (Art. 43) a Southbyo tophaceaePinguiculetum tejedensis. 


\section{Agradecimientos}

Dos revisores anónimos realizaron aportaciones valiosas que mejoraron el texto final. Trabajo financiado a cargo del proyecto de investigación AICO2019-003, de la Generalitat Valenciana.

\section{Esquema sintaxonómico}

Cl. Adiantetea Br.-BI. in Br.-BI., Roussine \& Nègre 1952

Ord. Adiantetalia capilli-veneris Br.-BI. ex Horvatić 1934

All. Pinguiculion longifoliae F. Casas 1970

Ass. Adianto capilli-veneris-Pinguiculetum longifoliae F. Casas 1970 [Pinguiculo longifoliae-Adiantetum capilli-veneris Rivas-Martínez, Báscones, T.E. Díaz, Fernández-González \& Loidi 1991]

Ass. Hyperico nummularii-Pinguiculetum coenocantabricae T.E. Díaz, Guerra \& Nieto 1982 corr. Rivas-Martínez, T.E. Díaz, F. Prieto, Loidi \& Penas 1984 [Hyperico-Pinguiculetum grandiflorae T.E. Díaz, Guerra \& Nieto 1982]

Ass. Adianto capilli-veneris-Pinguiculetum dertosensis (O. Bolós 1967) Deil ex M.B. Crespo, M.Á. Alonso \& Mart.-Azorín comb. \& stat. nov. [Eucladio-Adiantetum subass. pinguiculetosum dertosensis O. Bolòs 1967]

Ass. Eucladio verticillati-Pinguiculetum casperianae M.B. Crespo, M.Á. Alonso \& Mart.Azorín ass. nov. hoc loco

Ass. Adianto capilli-veneris-Pinguiculetum saetabensis M.B. Crespo, M.Á. Alonso, J.E. Oltra \& Mart.-Azorín 2019

Ass. Eucladio verticillati-Pinguiculetum mundi T.E. Díaz, Guerra \& Nieto 1982 corr. Asensi \& Díez Garretas in Rivas-Martínez, T.E. Díaz, FernándezGonzález, Izco, Loidi, Lousã \& Penas 2002 [Eucladio-Pinguiculetum vallisneriifoliae T.E. Díaz, Guerra \& Nieto 1982]

Ass. Pinguiculetum vallisneriifoliae Heywood ex Varo \& F. Casas 1970

Ass. Southbyo tophaceae-Pinguiculetum tejedensis Asensi \& Díez Garretas in RivasMartínez, T.E. Díaz, Fernández-González, Izco, Loidi, Lousã \& Penas 2002 corr. M.B. Crespo, M.Á. Alonso \& Mart.-Azorín, nom. corr. hoc loco

\section{Bibliografía}

Bolòs, O. de (1967). Comunidades vegetales de las comarcas próximas al litoral situadas entre los ríos Llobregat y Segura. Memorias de la Real Academia de Ciencias y Artes de Barcelona, 38(1), 1269.

Blanca, G. (2001). Pinguicula L. In J. Paiva et al. (Eds), Flora iberica, 14, 81-95. Madrid: Real Jardín Botánico, CSIC.

Blanca, G., Ruiz-Rejón, M. \& Zamora, R. (1999). Taxonomic revision of the genus Pinguicula $L$. in the Iberian Peninsula. Folia Geobotanica, 34, 337361. doi: https://doi.org/10.1007/BF02912819
Crespo, M.B., Alonso, M.Á., Oltra, J. \& MartínezAzorín, M. (2019a). Adianto-Pinguiculetum saetabensis (Pinguiculion longifoliae): una nueva asociación rupícola setabense. Flora Montiberica, 75, 8-10.

Crespo, M.B., Martínez-Azorín, M. \& Alonso, M.Á. (2019b). Morphological and molecular data support recognition of a new rupicolous species of Pinguicula (Lentibulariaceae) from the Iberian Peninsula. Plant Biosystems, 153, 77-87. doi: https://doi.org/10.1080/11263504.2018.1461702

Crespo, M.B., Martínez-Azorín, M., Alonso, M.Á. \& Sáez, L. (2020). Two new calcicolous species of Pinguicula sect. Pinguicula (Lentibulariaceae) growing on rocky habitats of the Iberian Peninsula. Phytotaxa, 456, 269-284.

Doi: https://doi.org/10.11646/phytotaxa.456.3.4

Deil, U. (1989). Adiantetea-Gesellschaften auf der Arabischen Halbinsel, Coenosyntaxa in dieser Klasse sowie allgemeine Uberlegungen zur Phylogenie von Pflanzengesellschaften. Flora, 182, 247-264. doi: https://doi.org/10.1016/S03672530(17)30414-0

Díaz, T.E., Guerra, J. \& Nieto, J.M. (1982). Contribución al conocimiento de la clase Adiantetea Br.BI. 1942 en la Península Ibérica. Anales del Jardín Botánico de Madrid, 38(2), 497-506.

Fernández Casas, J. (1970). Notas fitosociológicas breves, I. Ars Pharmaceutica, 11, 273-298.

Fernández Casas, J. \& Benito Alonso, J.L. (1999). Lectotipificación de la asociación AdiantoPinguiculetum longifoliae Fern. Casas 1970. Lazaroa, 20, 114.

Foucault, B. de (2015). Contribution au prodrome des végétations de France: les Adiantetea capilliveneris Braun-Blanq. ex Braun-Blanq., Roussine \& Nègre 1952. Acta Botanica Gallica. Botany Letters, 162, 375-403.

https://doi.org/10.1080/12538078.2015.1108868

Gómez Mercado, F. (2011). Vegetación y flora de la Sierra de Cazorla. Guineana, 17, 1-481.

Heywood, V.H. (1954). El concepto de asociación en las comunidades rupícolas. Anales del Jardín Botánico de Madrid, 11(2), 463-481.

Mota, J. F., Gómez Mercado, F. \& Valle, F. (1991). Rupicolous vegetation of the Betic ranges (south Spain). Vegetatio, 94, 101-113. Doi: https://doi.org/10.1007/BF00032624

Pérez Latorre, A.V., Navas Fernández, D., Gavira, O., Caballero, G. \& Cabezudo, B. (2004). Vegetación del Parque Natural de las Sierras de Tejeda, Almijara y Alhama (Málaga-Granada, España). Acta Botanica Malacitana, 29, 117-190.

Doi: https://doi.org/10.24310/abm.v29i0.7228

Rivas-Martínez, S. (2007). Mapa de series, geoseries y geopermaseries de vegetación de España [Memoria del Mapa de Vegetación Potencial de España. Parte 1]. Itinera Geobotanica, 17, 1-436.

Rivas-Martínez, S., Báscones, J.C., Díaz, T.E., Fernández-González, F. \& Loidi, J. (1991). Vegetación del Pirineo occidental y Navarra. Itinera Geobotanica, 5, 5-456.

Rivas-Martínez, S., Fernández-González, F., Loidi, J., 
Lousã, M. \& Penas, Á. (2001). Syntaxonomical checklist of vascular plant communities of Spain and Portugal to association level. Itinera Geobotanica, 14, 5-341.

Rivas-Martínez, S., Díaz, T.E., Fernández-González, F., Izco, J., Loidi, J., Lousã, M. \& Penas, Á. (2002). Vascular plant communities of Spain and Portugal. Addenda to the syntaxonomical checklist of 2001. Itinera Geobotanica, 15, 5-922.

Rivas-Martínez, S., Díaz, T.E., Fernández Prieto, J.A., Loidi, J. \& Penas, Á. (1984). La vegetación de la alta montaña cantábrica: Los Picos de Europa. León: Ediciones Leonesas. 295 pp.

Royo, F. (2006). Flora $i$ vegetació de les planes $i$ serres litorals compreses entre el riu Ebro i la serra d'Irta. Tesis Doctoral. Universitat de Barcelona. $1130 \mathrm{pp}$. https://bibdigital.rjb.csic.es/idurl/1/1526133

Sánchez Gómez, P., Güemes, J., Herranz, J.M., Fernández Jiménez, S., López Vélez, G. \&
Martínez Sánchez, J.J. (1997). Plantas vasculares endémicas, amenazadas 0 raras de la provincia de Albacete. Instituto de Estudios Albacetenses, Ser. I, no 92. Albacete: Excma. Diputación de Albacete. 223 pp.

Theurillat, J.-P., Willner, W., Fernández-González, F., Bültmann, H., Čarni, A., Gigante, D., Mucina, L. \& Weber, H. (2020). International Code of Phytosociological Nomenclature. $4^{\text {th }}$ edition. Applied Vegetation Science, 2020: e12491. Doi: https://doi.org/10.1111/avsc.12491

Varo, J. \& Fernández Casas, J. (1970). Notas sobre algunas comunidades béticas con Adiantum capillus-veneris. Ars Pharmaceutica, 11, 517-520.

Vera, J.B. (2015). Bases para la conservación de la flora protegida y hábitats prioritarios en el ámbito del sur de Albacete. Tesis Doctoral. Universidad de Murcia. $711 \mathrm{pp}$.

https://digitum.um.es/digitum/handle/10201/45965 